\title{
Monte Carlo simulation of survival for minority languages
}

\author{
Christian Schulze and Dietrich Stauffer \\ Institute for Theoretical Physics, Cologne University \\ D-50923 Köln, Euroland
}

October 17, 2018

e-mail: stauffer@thp.uni-koeln.de

\begin{abstract}
Our earlier language model is modified to allow for the survival of a minority language without higher status, just because of the pride of its speakers in their linguistic identity. An appendix studies the roughness of the interface for linguistic regions when one language conquers the whole territory.

Keywords: Language Competition, Monte Carlo, Quebec.
\end{abstract}

\section{Introduction}

Canada is a multicultural country with two official languages: English (majority) and French (minority). How is it possible that the minority language does not die out even though it has no higher prestige (status) there than the majority language English? We want to simulate this effect.

Abrams and Strogatz [1] introduced competition between languages in a model with only two languages, and there the minority language can survive only if it has a higher status (prestige) than the majority language. With some modification also coexistence is possible [2] but still a status advantage is needed. Other aspects of language competition were recently reviewed by us [3]. The present work uses our previous multi-language model [4] to explain a stable survival of one minority language without an overall status advantage. 


\section{Model}

The model [4 is a variant and generalisation of our earlier bit-string model [3] and uses Potts variables $q=1,2, \ldots, Q$ instead of merely bits. Each language or grammar is characterised by $F$ features, and each of these features is an integer between 1 and $Q$. Thus we have $Q^{F}$ possible languages or grammars.

People sit on $L \times L$ square lattices, one person and one language per lattice site. Initially each person speaks a randomly selected language. Then, at each iteration, three processes mutation, transfer and flight happen with probabilities $p, q, r$ :

Each of the $F$ features is mutated with probability $p$. If such a mutation happens, then with probability $1-q$ a random integer between 1 and $Q$ is selected as the new value for this feature, while with probability $q$ one of the four lattice neighbours is selected randomly and its value for this feature is transferred to become the value of this feature for the mutated language. If the language is spoken by a fraction $x$ of the whole population, then with probability $r(1-x)^{2}$ the simulated person switches its language to that of a randomly selected lattice neighbour. (In our earlier version [4 this flight away from a small language was made to the language of a person randomly selected from the whole lattice instead of from the four neighbours; in that case we do not get coherent language regions.) Usually, $r=0.9$.

As in earlier versions, this model gives a sharp phase transition where the equilibrium fraction of people speaking the largest language jumps from a small value to nearly unity, that means the population moves from fragmentation (into many languages) to dominance (of one language).

We now assume that the speakers of one particular language, which may be identified with French in case of Canada, start to defend their language as soon as the largest llanguage (English) has attracted more than half of the population. From then on the flight from French to other languages no longer takes place, that means $r=0$ for all French speakers. The other languages (native population, other European immigrants, ...) continue as usual towards near-extinction.

\section{Results}

Fig.1 shows the growth of the majority language, and Fig.1b the variation of the special language (i.e. French); initially both are spoken by about 0.4 percent of the population. We see that after slightly more than 1000 iterations, when English reaches the 50 percent threshold, the French language recovers from its previous losses and attracts even more people than initially, though it remains less widespread than English (except perhaps for very long times). 

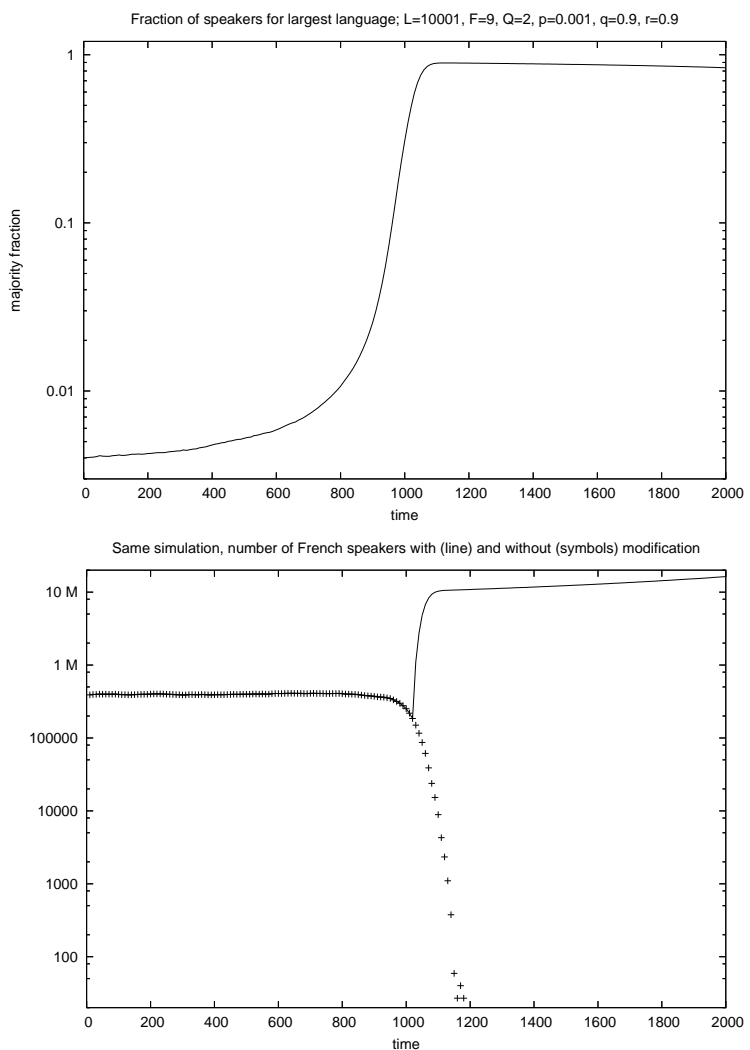

Figure 1: Time variation of "English" (top) and "French" (bottom) with 100 million people, with and without the modification regarding flight from French.

Fig. 2 shows for a smaller lattice with $10^{5}$ instead of $10^{8}$ sites that French speaking people form geographically connected clusters, both before and after English surpasses the 50 percent threshold at $\mathrm{t}=244$. The French clusters before this threshold time are much smaller than afterwards.

These simulations were made for small mutation rates $p=0.001$, where dominance always emerges independent of $q$. For larger $p$, dominance is possible only for large $q$, and Fig. 3 shows the phase diagram: In the left part (small $p$ or large q) fragmentation switches over to dominance while in the right part (large $p$ or small $q$ ) fragmentation stays during the observation time of $10^{4}$ iterations. (Errors are about 0.02 in $q$ at fixed $p$ and 0.001 in $p$ at fixed $q$. ) We see little qualitative dependence on these parameters, but the more possible languages we simulate (the higher $F$ or $Q$ is), the more difficult is the emergence of dominance from the initial 

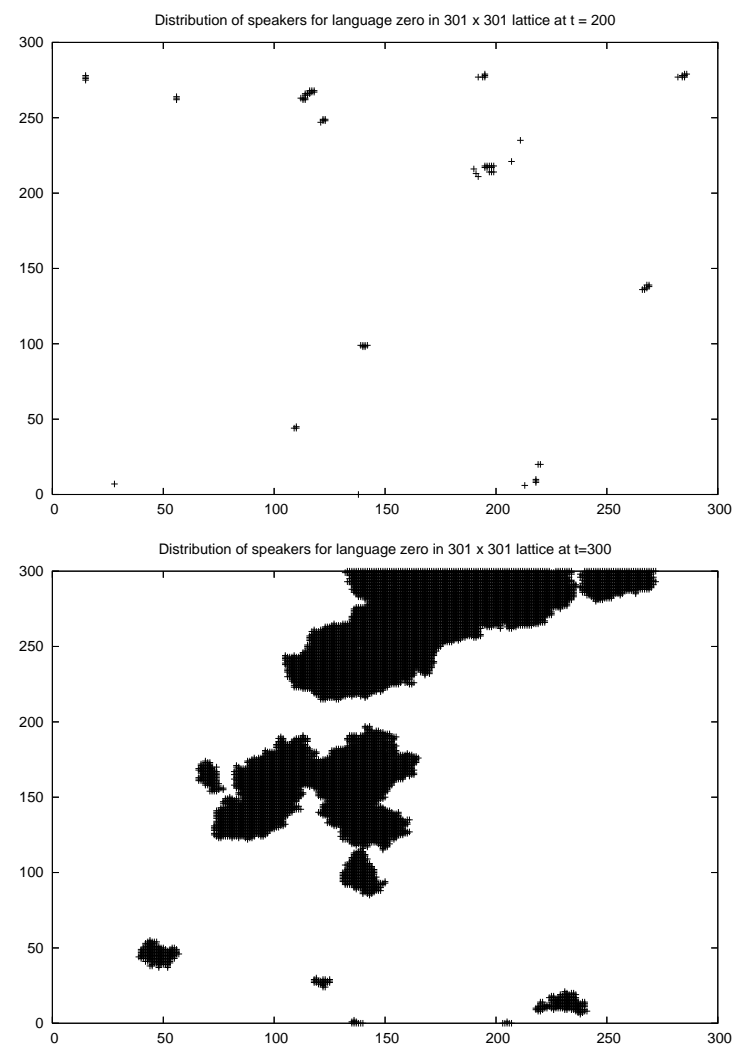

Figure 2: Clusters of French-speaking people before (top) and after (bottom) English became the dominating language and French speakers no longer left their language. As in Fig.1: $F=8, Q=2, p=0.001, q=0.9, r=0.9$.

fragmentation. Fig.4 shows unusually large finite-size effects: Only for $L>82$ a transition to dominance could be seen at $p=0.01$; nevertheless a finite transition point $q_{c} \sim 0.76$ seems plausible for infinitely large lattices. (As a function of observation time $t$, at $L=2, F=8, Q=2, p=0.01, r=0.9$ the threshold $q_{c}$ diminished from 0.65 and 0.56 to 0.52 and 0.48 for $t=10^{3}, 10^{4}, 10^{5}, 10^{6}$.)

One might envisage a situation when the use of French dies out even after the flight away from French is stopped. This happened for our parameters if French had died out already before the flight from French was stopped. Such a situation is nearly unavoidable for large $F$, large $Q$ and small $L$, when there are much more possible languages $Q^{F}$ than people $L^{2}$. 


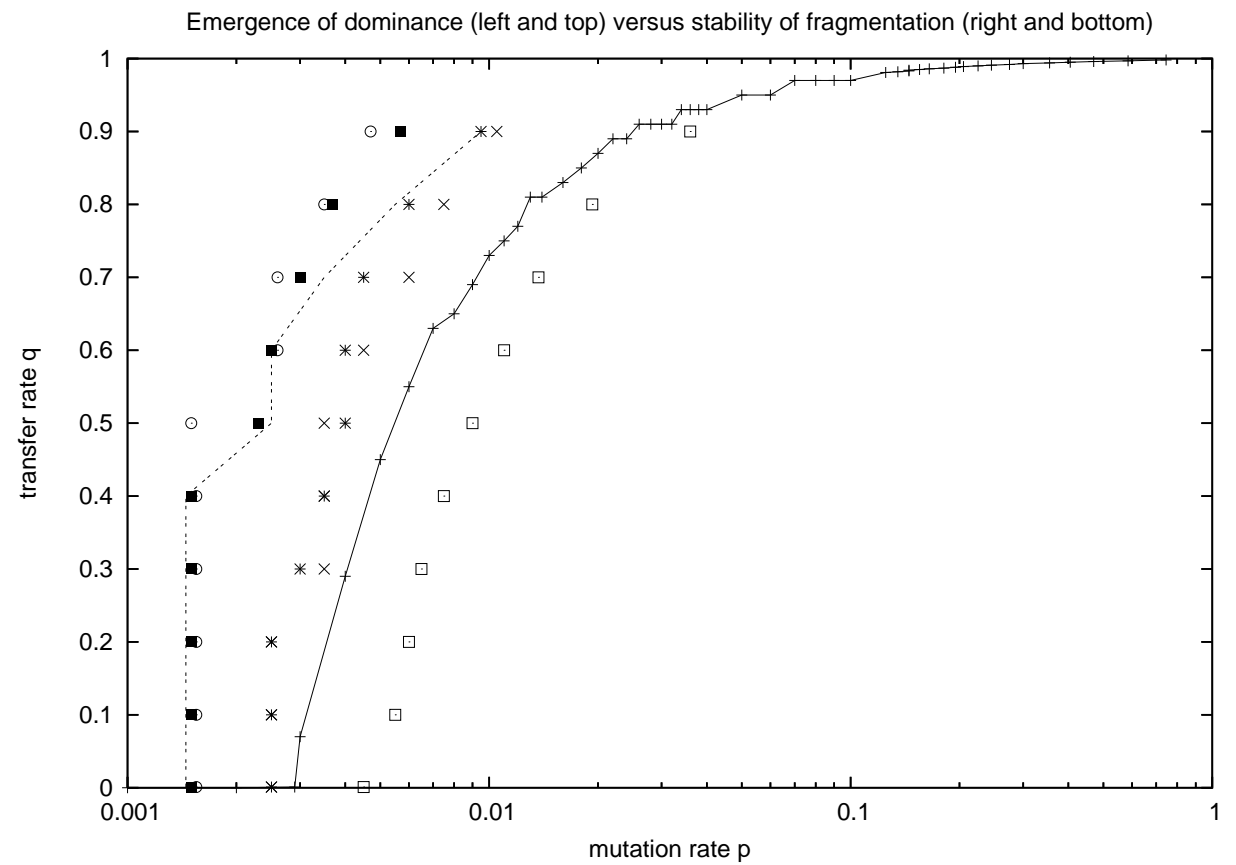

Figure 3: Phase diagram for $L=201, t=10,000, r=0.9$. To the left we get dominance to the right we stay at fragmentation. $(F, Q)=(8,2$; empty square), ( 8,$3 ; \mathrm{x}),(8,5 ; *),(16,2$; full square), (16,3; empty circles) from right to left. The lines have $F=8, Q=2$ for $L=1001$ (one sample) instead of 201 (four samples), with the usual $r=0.9$ for the long line and $r=0.5$ for the short line.

\section{Conclusion}

We modified our previous multi-language model by allowing flight away from small languages only to languages spoken by a lattice neighbour, and by switching off this flight for one particular language (French) once the dominating language (English) surpassed a threshold of half of the population. As a result, the French language did not only survive (if it did not become extinct before) but even could attract more speakers than at the initial random distribution of the population among all possible languages. 


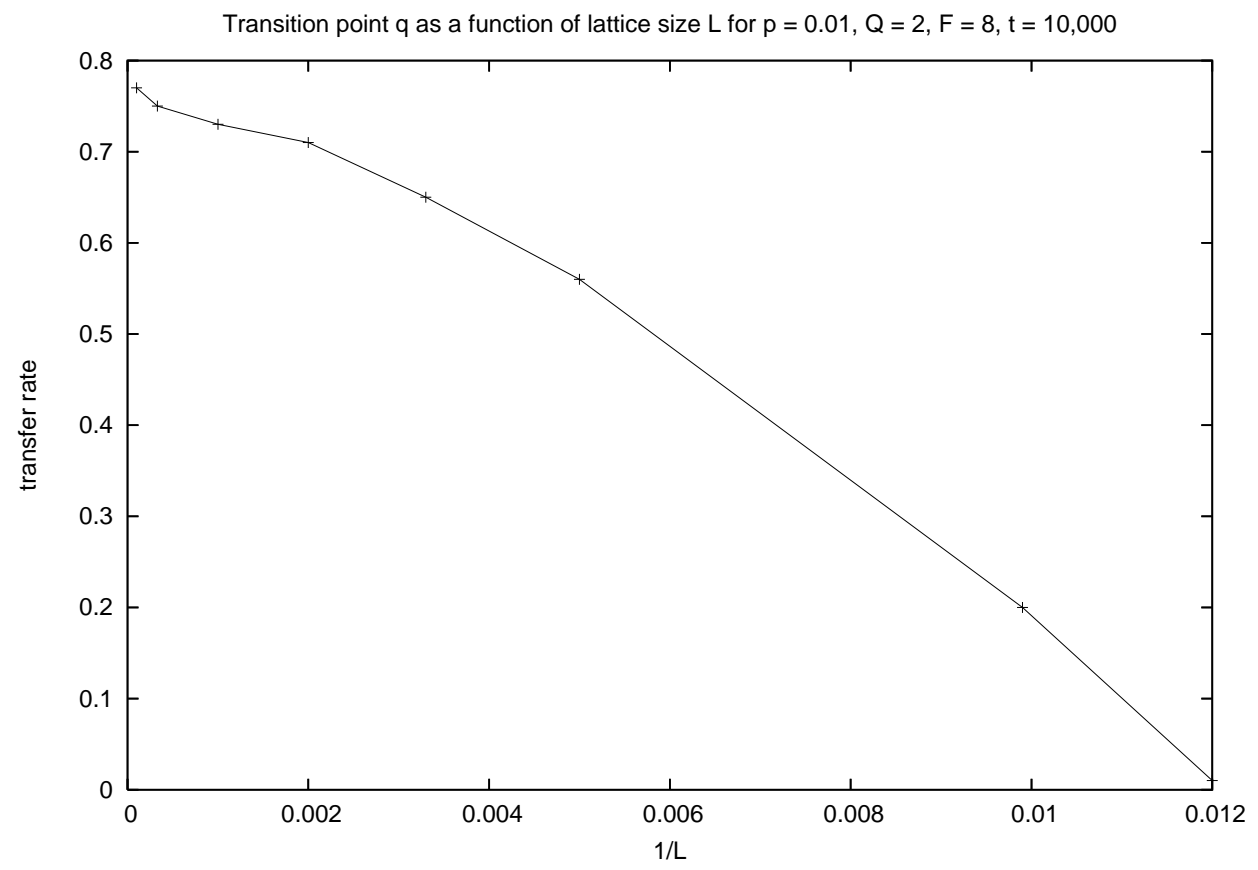

Figure 4: Strong size effects for transition point $q$ with $83 \leq L \leq 10001$.

\section{Appendix}

The irregular clusters of language domains in Fig.2 are not suited to study surface roughening, familiar to physicists since decades. To study the structure of the interface between French and the other languages we thus started with the bottom five percent of the lattice speaking only French, and omitted the rule that the French stop switching to English once more than half of the population speaks English. Because of our initialisation with five percent, French is the dominating language anyhow and the no-switch condition is never fulfilled. The simulation now corresponds more to the boundary between a conquering language and native different languages, like in Quebec three centuries ago. Fig.5 shown an intermediate example of the growth of French. At the end the border merges with the upper lattice line and nearly everybody speaks French.

We now define a width $W$ of the interface region between black and white in Fig. 5 by determining for each column $k$ the highest black place $i_{k}$ where French is spoken. Then our width is the mean square line number $i$ :

$$
W^{2}=\sum_{k}\left(i_{k}-<i>\right)^{2} / H ; \quad<i>,=\sum_{k} i_{k} / H
$$




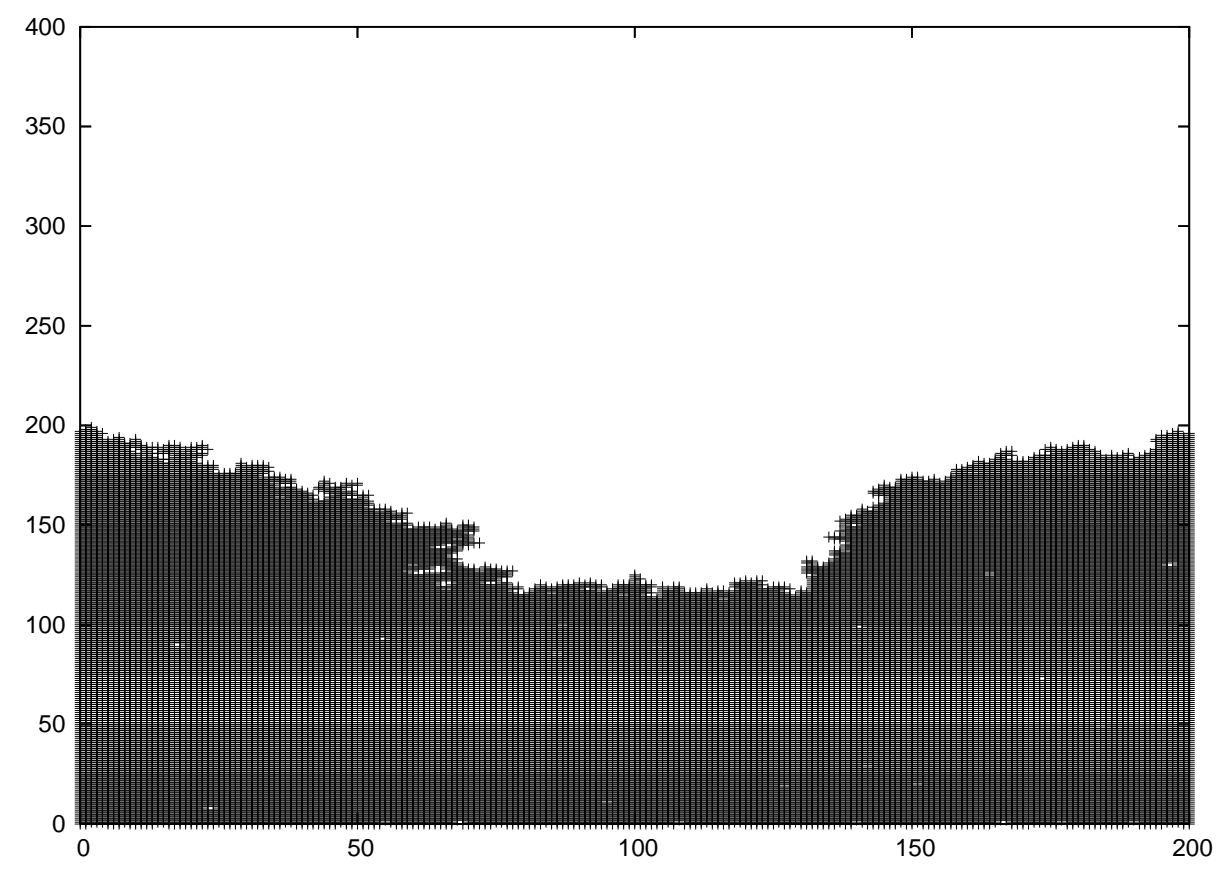

Figure 5: Growth of the region where the dominating language is spoken, at intermediate times. At the end nearly everybody speaks that language

where $H$ (up to 800) is the height of the $L \times H$ lattice. Fig.6 shows for various parameters the variation of $W$ with $L, H, q$; that with $q$ is quite small. For our standard case $Q=2, F=8$ we found a linear increase of $W$ with time until the finite height hinders further growth, Fig.6a; for the other parameters $Q=3$ and 5 , and $F=16$, the $\log$-log plots Fig.6b-d are less clear. Perhaps the First Nations in Quebec were not interested in the exponents of the Kardar-Parisi-Zhang equation.

\section{References}

[1] D.M. Abrams and S.H. Strogatz, Nature 424, 900 (2003)

[2] J.P. Pinasco and L. Romanelli, Physica A 361, 355 (2006)

[3] B.K. Chakrabarti, A. Chakraborti and A. Chatterjee, eds., it Econophysics and Sociophysics: Trends and Perspectives, Wiley-VCH, Weinheim 2006, chapter 11 . 

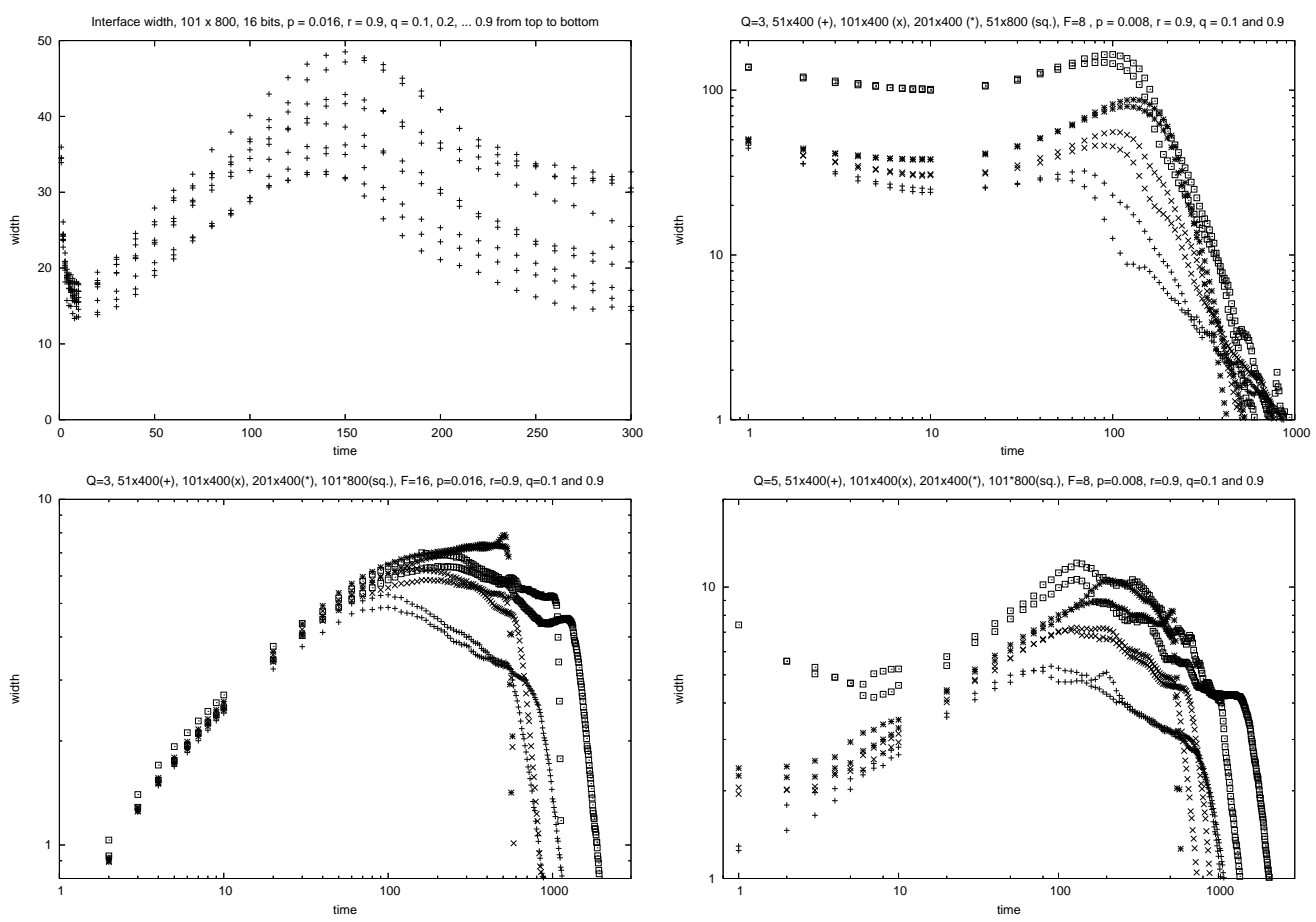

Figure 6: Width versus time for $Q=2$ (part a), $Q=3$ (parts b,c) and $Q=5$ part d), with $F=8$ (parts b,d) and 16 (parts a,c). For $Q=2, F=8$ (not shown) the width decays rapidly to zero.

[4] C. Schulze and D. Stauffer, Computing in Science and Engineering 8, 86 (May/June 2006) 